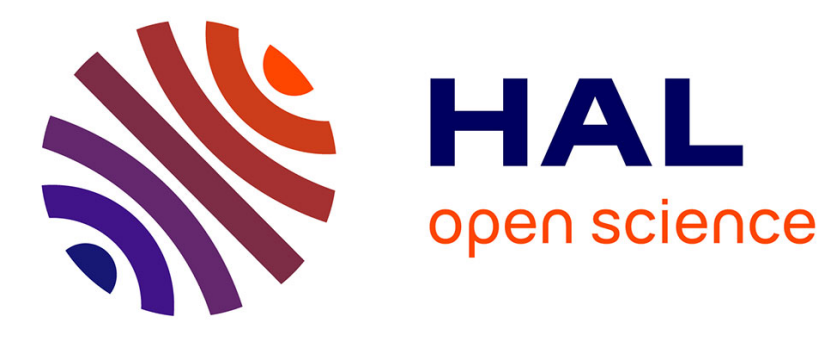

\title{
Intelligent Information Processing X
}

\author{
Zhongzhi Shi, Sunil Vadera, Elizabeth Chang
}

\section{To cite this version:}

Zhongzhi Shi, Sunil Vadera, Elizabeth Chang. Intelligent Information Processing X: 11th IFIP TC 12 International Conference, IIP 2020, Hangzhou, China, July 3-6, 2020, Proceedings. Springer International Publishing, AICT-581, 2020, IFIP Advances in Information and Communication Technology, 978-3-030-46930-6. 10.1007/978-3-030-46931-3 . hal-03456961

\section{HAL Id: hal-03456961 \\ https://hal.inria.fr/hal-03456961}

Submitted on 30 Nov 2021

HAL is a multi-disciplinary open access archive for the deposit and dissemination of scientific research documents, whether they are published or not. The documents may come from teaching and research institutions in France or abroad, or from public or private research centers.
L'archive ouverte pluridisciplinaire HAL, est destinée au dépôt et à la diffusion de documents scientifiques de niveau recherche, publiés ou non, émanant des établissements d'enseignement et de recherche français ou étrangers, des laboratoires publics ou privés. 


\section{IFIP Advances in Information and Communication Technology}

\section{Editor-in-Chief}

Kai Rannenberg, Goethe University Frankfurt, Germany

\section{Editorial Board Members}

TC 1 - Foundations of Computer Science

Luís Soares Barbosa (D), University of Minho, Braga, Portugal

TC 2 - Software: Theory and Practice

Michael Goedicke, University of Duisburg-Essen, Germany

TC 3 - Education

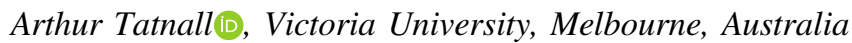

TC 5 - Information Technology Applications

Erich J. Neuhold, University of Vienna, Austria

TC 6 - Communication Systems

Burkhard Stiller, University of Zurich, Zürich, Switzerland

TC 7 - System Modeling and Optimization

Fredi Tröltzsch, TU Berlin, Germany

TC 8 - Information Systems

Jan Pries-Heje, Roskilde University, Denmark

TC 9 - ICT and Society

David Kreps (iD, University of Salford, Greater Manchester, UK

TC 10 - Computer Systems Technology

Ricardo Reis(D), Federal University of Rio Grande do Sul, Porto Alegre, Brazil

TC 11 - Security and Privacy Protection in Information Processing Systems

Steven Furnell $\mathbb{D}$, Plymouth University, UK

TC 12 - Artificial Intelligence

Eunika Mercier-Laurent ${ }^{\mathbb{D}}$, University of Reims Champagne-Ardenne, Reims, France

TC 13 - Human-Computer Interaction

Marco Winckler $\mathbb{D}$, University of Nice Sophia Antipolis, France

TC 14 - Entertainment Computing

Rainer Malaka, University of Bremen, Germany 


\section{IFIP - The International Federation for Information Processing}

IFIP was founded in 1960 under the auspices of UNESCO, following the first World Computer Congress held in Paris the previous year. A federation for societies working in information processing, IFIP's aim is two-fold: to support information processing in the countries of its members and to encourage technology transfer to developing nations. As its mission statement clearly states:

IFIP is the global non-profit federation of societies of ICT professionals that aims at achieving a worldwide professional and socially responsible development and application of information and communication technologies.

IFIP is a non-profit-making organization, run almost solely by 2500 volunteers. It operates through a number of technical committees and working groups, which organize events and publications. IFIP's events range from large international open conferences to working conferences and local seminars.

The flagship event is the IFIP World Computer Congress, at which both invited and contributed papers are presented. Contributed papers are rigorously refereed and the rejection rate is high.

As with the Congress, participation in the open conferences is open to all and papers may be invited or submitted. Again, submitted papers are stringently refereed.

The working conferences are structured differently. They are usually run by a working group and attendance is generally smaller and occasionally by invitation only. Their purpose is to create an atmosphere conducive to innovation and development. Refereeing is also rigorous and papers are subjected to extensive group discussion.

Publications arising from IFIP events vary. The papers presented at the IFIP World Computer Congress and at open conferences are published as conference proceedings, while the results of the working conferences are often published as collections of selected and edited papers.

IFIP distinguishes three types of institutional membership: Country Representative Members, Members at Large, and Associate Members. The type of organization that can apply for membership is a wide variety and includes national or international societies of individual computer scientists/ICT professionals, associations or federations of such societies, government institutions/government related organizations, national or international research institutes or consortia, universities, academies of sciences, companies, national or international associations or federations of companies.

More information about this series at http://www.springer.com/series/6102 
Zhongzhi Shi · Sunil Vadera ·

Elizabeth Chang (Eds.)

\section{Intelligent \\ Information}

Processing X

11th IFIP TC 12 International Conference, IIP 2020 Hangzhou, China, July 3-6, 2020

Proceedings

粤 Springer 


\section{Editors}

Zhongzhi Shi

Institute of Computing Technology

Chinese Academy of Sciences

Beijing, China

Elizabeth Chang

Australian Defence Force Academy

UNSW Canberra

Canberra, ACT, Australia

\author{
Sunil Vadera \\ University of Salford \\ Manchester, UK
}

\author{
ISSN 1868-4238 ISSN 1868-422X (electronic) \\ IFIP Advances in Information and Communication Technology \\ ISBN 978-3-030-46930-6 ISBN 978-3-030-46931-3 (eBook) \\ https://doi.org/10.1007/978-3-030-46931-3
}

(C) IFIP International Federation for Information Processing 2020

This work is subject to copyright. All rights are reserved by the Publisher, whether the whole or part of the material is concerned, specifically the rights of translation, reprinting, reuse of illustrations, recitation, broadcasting, reproduction on microfilms or in any other physical way, and transmission or information storage and retrieval, electronic adaptation, computer software, or by similar or dissimilar methodology now known or hereafter developed.

The use of general descriptive names, registered names, trademarks, service marks, etc. in this publication does not imply, even in the absence of a specific statement, that such names are exempt from the relevant protective laws and regulations and therefore free for general use.

The publisher, the authors and the editors are safe to assume that the advice and information in this book are believed to be true and accurate at the date of publication. Neither the publisher nor the authors or the editors give a warranty, expressed or implied, with respect to the material contained herein or for any errors or omissions that may have been made. The publisher remains neutral with regard to jurisdictional claims in published maps and institutional affiliations.

This Springer imprint is published by the registered company Springer Nature Switzerland AG The registered company address is: Gewerbestrasse 11, 6330 Cham, Switzerland 


\section{Preface}

This volume comprises of papers presented at the 11th IFIP International Conference on Intelligent Information Processing (IIP 2020). As the world proceeds quickly into the Information Age, it encounters both successes and challenges, and it is well recognized that intelligent information processing provides the key to solve many challenges in the Information Age. Intelligent information processing supports the most advanced techniques that are able to change human life and the world. However, the path to success is never a straight one. Every new technology brings with it many challenging problems, and researchers are in great demand to tackle these. This conference provides a forum for engineers and scientists from research institutes, universities, and industries to report and discuss their latest research progresses in all aspects of intelligent information processing.

We received more than 36 papers, of which 24 papers are included in this program as regular papers and 5 as short papers. All papers submitted were reviewed by three reviewers. We are grateful for the dedicated work of both authors and reviewers.

A conference such as this cannot succeed without help from many individuals who contributed their valuable time and expertise. We want to express our sincere gratitude to the Program Committee members and referees, who invested many hours into reviews and deliberations. They provided detailed and constructive review comments that significantly improved the quality of the papers included in these proceedings.

We are very grateful to have the sponsorship of the following organizations: IFIP TC12, Key Laboratory of Brain Machine Collaborative Intelligence of Zhejiang Province at Hangzhou Dianzi University and Institute of Computing Technology, Chinese Academy of Sciences. We specially thank Wanzeng Kong, Jianhai Zhang and Yang Song for organizing the conference and Xuanyu Jin, Wenfen Ling for carefully checking the proceedings.

Finally, we hope you find this volume inspiring and informative. We wish that the research results reported in these proceedings will lead to exciting new findings in the years to come.

March 2020

Zhongzhi Shi

Sunil Vadera

Elizabeth Chang 


\section{Organization}

\section{General Chairs}
U. Furbach, Germany
B. Goertzel, USA
Q. Wu, China

\section{Program Chairs}

Z. Shi, China

S. Vadera, UK

E. Chang, Australia

\section{Local Organizing Committee}

Wanzeng Kong (Chair), China

Jianhai Zhang (Co-chair), China

Yang Song (General Secretary), China

\section{Program Committee}
B. An, Singapore
E. Chang, Australia
L. Chang, China
S. Ding, China
Q. Dou, China
E. Ehlers, South Africa
J. Feng, China
Y. Feng, China
Z. Feng, China
U. Furbach, Germany
Y. Gao, China
Q. He, China
Z. Huang, The Netherlands
S. Jiang, China
F. Kong, China
H. Leung, Hong Kong

G. Li, Australia

Q. Li, China

H. Leung, Hong Kong

H. Ma, China

J. Ma, China

S. Ma, China

X. Mao, China

L. Maglogiannis, Greece

Z. Meng, China

E. Mercier-Laurent, France

P. Novias, Portugal

M. Owoc, Poland

Vasile Palade, UK

G. Pan, China

L. Qin, China

A. Rafea, Egypt
Y. H. Shi, China

Z. P. Shi, China

K. Shimohara, Japan

A. Skowron, Poland

I. Timm, Germany

G. Wang, China

P. Wang, USA

Y. Xu, Australia

H. Xiong, USA

Y. Yao, Canada

B. Zhang, China

J. Zhang, China

S. Zhang, China

Z. Zhang, China

J. Zhou, China

Z. Zhou, China

F. Zhuang, China 


\section{Abstracts of Keynotes and Invited Presentations}




\title{
Tensor Networks and Their Applications in Image and Time Series Reconstruction and Prediction
}

\author{
Andrzej Cichocki \\ Skolkovo Institute of Science and Technology (SKOLTECH), Russia
}

\begin{abstract}
Tensor factorizations/decompositions and their extensions tensor networks have become emerging techniques in AI and machine learning methods. Tensor networks have already been successfully used over a number of applied areas in machine learning, including deep neural networks, generalized regression, classification, clustering, support tensor machines (STM), tensor canonical correlation analysis (TCCA), higher order partial least squares (HOPLS), multilinear independent component analysis (MLICA), Non-negative Tensor Factorization (NTF), multiway robust PCA (MRPCA), and Higher order SVD.

In this talk, we will start with a brief overview of these applications, and special emphasis will be given to emerging applications in image reconstructions and enhancement, and higher order time series forecasting (TSF).
\end{abstract}




\title{
Behavior Based Artificial Intelligence from the Perspective of Automatic Control
}

\author{
Fuchun Sun \\ Tsinghua University, China
}

\begin{abstract}
In the development of human society, the invention and use of machinery is an important sign of human beings entering into industrialization, and machinery becomes an independent actuator, which realizes the separation of executor and action instruction sender from us as humans. Furthermore, the emergence of artificial intelligence makes it possible for a machine to become a sender of instructions through interactions with the environment and humans, and as a result, the sender and executor of the instruction are unified as the machine itself. This report systematically analyzes behavior-based artificial intelligence from evolution of automatic control. Then, taking into account the topic "how to sense like a human being," the talk puts forward the framework of robot active perception, introduces the main achievements of the team in visual multi-target detection, visual tactile representation, multimodal fusion, and developmental learning, and tackles "how to operate like a human being" as a problem, providing the main achievements of the team in learning smart operation skills such as active imitation learning and preference learning achievements. Next, taking into account the topic "how to make decision like a human being," some new points are given in terms of micro-size intelligence. Finally, the development trend of robot dexterous operation skill learning under the condition of incomplete information is given.
\end{abstract}




\title{
Tensor Network Representations in Machine Learning
}

\author{
Qibin Zhao
}

RIKEN AIP, Japan

\begin{abstract}
Tensor networks are factorizations of very large tensors into networks of smaller tensors, it is shown to be a general extension of typical tensor decomposition to high dimensional case. Recently, tensor networks are also increasingly finding applications in machine learning such as model compression or acceleration of computations. In this talk, I will first present the general concept of tensor network related research in machine learning, and then introduce our studies on fundamental tensor network model, algorithm, and applications. In particular, the tensor ring decomposition model is introduced and shown to be a powerful and efficient representation. In addition, we will present recent progresses on how tensor networks can be employed to solve challenging problems in tensor completion, multi-task learning, and multi-modal learning.
\end{abstract}




\title{
Uncertainty Between Prediction and Interpretability
}

\author{
Junping Zhang \\ Fudan University, China
}

\begin{abstract}
The goal of artificial intelligence is to approximate human's minds, making a computer indistinguishable with human beings. In the era of deep learning, we see the prediction performance of computer program is almost superior to that of human. However, the higher the prediction performance, the farther the distance that we reach the real intelligence. In this talk, I will discuss this issue from the view of the uncertainty between prediction and interpretability, and mention some possible direction based on the uncertainty principle.
\end{abstract}




\title{
Neuromorphic Computing Approach to Auditory and Visual Perception
}

\author{
Huajin Tang \\ Zhejiang University, China
}

\begin{abstract}
Neuromorphic computing has become an important methodology to emulate brain style intelligence. This talk will present an overview of neuromorphic approach to auditory and visual perception and highlight the recent state of the art.

In recent years neuromorphic computing has become an important methodology to emulate brain style intelligence. There has been rapid progress in computational theory, learning algorithms, signal processing, and circuit design and implementation. By using neural spikes to represent the outputs of sensors and for communication between computing blocks, and using spike timing based learning algorithms, neuromorphic computational models and hardware have achieved promising real-time learning performance. This talk will start by introducing the computational principles and architecture found in neural systems, and present a new theme of neuromorphic approach to auditory and visual perception. In neuromorphic vision, the model extracts temporal features embedded in address-event representation (AER) data and discriminates different objects by using spiking neural networks (SNNs). We use multispike encoding to extract temporal features contained in the AER data. These temporal patterns are then learned through a spiking neural network using a supervised learning algorithm. The presented model is consistently implemented in a temporal learning framework, where the precise timing of spikes is considered in the feature-encoding and learning process. For neuromorphic auditor perception, I will introduce a robust multi-label classification model based on deep spiking neural networks to handle multi-pitch estimation tasks. In this model, we propose a novel biological spiking coding method that fits the expression of musical signals. This coding method can encode time, frequency, and intensity information into spatiotemporal spike trains. And the spatio-temporal credit assignment (STCA) algorithm is used to train deep spiking neural networks.
\end{abstract}




\title{
Neuromusicology with Machine Learning - How Human Understands Music
}

\author{
Toshihisa Tanaka
}

Tokyo University of Agriculture and Technology, Japan

\begin{abstract}
This talk addresses my recent neurophysiological studies of music cognition. I will talk about the neural entrainment to the familiarity of a listener with music using both the electroencephalogram (EEG) signals and the music. It is shown that the cross-correlation between EEG and the music when listening to unfamiliar music is significantly stronger than that when listening to familiar music. Moreover, the familiarity of music can be classified by machine learning techniques such as neural networks and support vector machines.
\end{abstract}




\title{
Intelligent Analysis of Brain Imaging for Early Diagnosis of Brain Diseases
}

\author{
Daoqiang Zhang \\ Nanjing University of Aeronautics and Astronautics, China
}

\begin{abstract}
In recent years, the brain research projects have received considerable public and governmental attention worldwide. Brain imaging technique is an important tool for brain science research. However, due to the high-dimensional, multi-modality, heterogenous, and time-variant characteristics of brain images, it is very challenging to develop both efficient and effective methods for brain image analysis. In this talk, I will introduce our recent works on intelligent methods of brain imaging, based on machine learning techniques. Specifically, this talk will cover the topics including multi-modal brain image fusion and classification, image genomic association analysis, functional alignment and brain network analysis, as well as their applications in early diagnosis of brain disease and brain decoding.
\end{abstract}




\title{
Data-Driven Security Analysis of Machine Learning Systems
}

\author{
Chao Shen \\ Xi'an Jiaotong University, China
}

\begin{abstract}
Human society is witnessing a wave of machine learning (ML) driven by deep learning techniques, bringing a technological revolution for human production and life. In some specific fields, ML has achieved or even surpassed human-level performance. However, most previous ML theories have not considered the open and even adversarial environments, and the security and privacy issues are gradually rising. Besides of insecure code implementations, biased models, adversarial examples, sensor spoofing can also lead to security risks, which are hard to be discovered by traditional security analysis tools. This talk reviews previous works on ML system security and privacy, revealing potential security and privacy risks. Firstly, we introduce a threat model of ML systems, including attack surfaces, attack capabilities, and attack goals. Secondly, we analyze security risks and countermeasures in terms of four critical components in ML systems: data input (sensor), data preprocessing, ML model, and output. Finally, we discuss future research trends on the security of ML systems. The aim is to rise the attention of the computer security society and the ML society on security and privacy of ML systems, and so that they can work together to unlock ML's potential to build a brighter future.
\end{abstract}




\section{Contents}

\section{Machine Learning}

A Salient Object Detection Algorithm Based on Region Merging

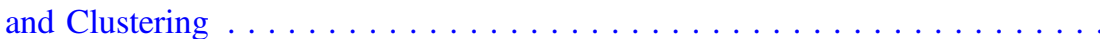

Weiyi Wei, Yijing Yang, Wanru Wang, Xiufeng Zhao, and Huifang Ma

Link-Based Cluster Ensemble Method for Improved

Meta-clustering Algorithm . . . . . . . . . . . . . . . . . . .

Changlong Shao and Shifei Ding

Large-Scale Spectral Clustering with Stochastic Nyström Approximation . . . . Hongjie Jia, Liangjun Wang, and Heping Song

Feature Selection Algorithm Based on Multi Strategy

Grey Wolf Optimizer. . . . . . . . . . . . . . . . . . . . . . . . . .

Guangyue Zhou, Kewen Li, Guoqiang Wan, and Hongtu Ji

A Novel Fuzzy C-means Clustering Algorithm Based on Local Density. . . . . Jian-jun Liu and Jian-cong Fan

A Novel Method to Solve the Separation Problem of LDA . . . . . . . . . . Meng Zhang, Wei Li, and Bo Zhang

Multi-label Classification of Short Text Based on Similarity Graph and Restart Random Walk Model . . . . . . . . . . . . . . . . . . . . . . .

Xiaohong Li, Fanyi Yang, Yuyin Ma, and Huifang Ma

Environmental Parameters Analysis and Power Prediction for Photovoltaic

Power Generation Based on Ensembles of Decision Trees . . . . . . . . . . . .

Shuai Zhang, Hongwei Dai, Aizhou Yang, and Zhongzhi Shi

The Conjugate Entangled Manifold of Space-Time Induced by the Law of Unity of Contradiction . . . . . . . . . . . . . . . . .

Jiali Feng and Jingjuan Feng

Similarity Evaluation with Wikipedia Features . . . . . . . . . . . . .

Shahbaz Wasti, Jawad Hussain, Guangjiang Huang,

and Yuncheng Jiang 


\section{Multi-Agent System}

Adaptive Game AI-Based Dynamic Difficulty Scaling via the Symbiotic

Game Agent. . . . . . . . . . . . . . . . . . . . . . . . . .

Siphesihle Philezwini Sithungu and Elizabeth Marie Ehlers

\section{Recommendation System}

Scientific Paper Recommendation Using Author's Dual

Role Citation Relationship . . . . . . . . . . . . . . . . . . .

Donglin Hu, Huifang Ma, Yuhang Liu, and Xiangchun He

A Genetic Algorithm for Travel Itinerary Recommendation with Mandatory Points-of-Interest . . . . . . . . . . . . . . . . .

Phatpicha Yochum, Liang Chang, Tianlong Gu, Manli Zhu, and Hongliang Chen

\section{Social Computing}

Stochastic Blockmodels Meets Overlapping Community Detection . . . . . . . .

Qiqi Zhao, Huifang Ma, Zhixin Li, and Lijun Guo

Overlapping Community Detection Combining Topological Potential and Trust Value of Nodes . . . . . . . . . . . . . . . . . . . . . . .

Xiaohong Li, Weiying Kong, Weiyi Wei, Enli Fu, and Huifang $\mathrm{Ma}$

\section{Brain Computer Integration}

Coarse-to-Fine Classification with Phase Synchronization and Common Spatial Pattern for Motor Imagery-Based BCI. . . . . . . . . . . . . . . . . . Wenfen Ling, Feipeng Xu, Qiaonan Fan, Yong Peng, and Wanzeng Kong

Ballistocardiogram Artifact Removal for Concurrent EEG-fMRI Recordings Using Blind Source Separation Based on Dictionary Learning . . . 180 Yuxi Liu, Jianhai Zhang, Bohui Zhang, and Wanzeng Kong

Comparison of Machine Learning and Deep Learning Approaches for Decoding Brain Computer Interface: An fNIRS Study . . . . . . . . . . . . . Jiahao Lu, Hongjie Yan, Chunqi Chang, and Nizhuan Wang 


\section{Pattern Recognition}

Phase Plane Analysis of Traffic Flow Evolution Based on a Macroscopic Traffic Flow Model. . . . . . . . . . . . . . . . . . . .

WenHuan Ai, Tao Xing, YuHang Su, DaWei Liu, and Huifang Ma

Phase Plane Analysis of Traffic Phenomena with Different Input and Output Conditions.

WenHuan Ai, YuHang Su, Tao Xing, DaWei Liu, and Huifang Ma

Bird Detection on Transmission Lines Based on DC-YOLO Model. . . . . . . .

Cong Zou and Yong-quan Liang

Research on Customer Credit Scoring Model Based

on Bank Credit Card

Maoguang Wang and Hang Yang

Analysis of the Stability and Solitary Waves for the Car-Following Model on Two Lanes . . . . . . . . . . . . . . . . . . . . . . . . . . . . . . . .

WenHuan Ai, Tao Xing, YuHang Su, DaWei Liu, and Huifang Ma

Queue Length Estimation Based Defence Against Data Poisoning Attack for Traffic Signal Control. . . . . . . . . . . . . . . . . . . .

Xu Gao, Jiqiang Liu, Yike Li, Xiaojin Wang, YingXiao Xiang,

Endong Tong, Wenjia Niu, and Zhen Han

A Method of Style Transfer for Chinese Painting . . . . . . . . . . . . . .

Cunjian Chen

Speech Triggered Mobility Support and Privacy . . . . . . . . . . . . .

Michael Zipperle, Marius Becherer, and Achim Karduck

\section{Computer Vision and Image Understanding}

Explaining Color Evolution, Color Blindness, and Color Recognition by the Decoding Model of Color Vision . . . . . . . . . . . .

Chenguang $\mathrm{Lu}$

A Content-Based Deep Hybrid Approach with Segmented Max-Pooling . . . . Dapeng Zhang, Liu Yajun, and Jiancheng Liu

Image Caption Combined with GAN Training Method . . . . . . . . . . .

Zeqin Huang and Zhongzhi Shi 